\title{
„Innovationen in der Bildgebung leisten einen wichtigen Beitrag für die Nachhaltigkeit des Gesundheitssystems"
}

Innovationen bringen die Medizin und die Radiologie voran, in beiden Bereichen werden die Innovationszyklen immer kürzer. Wie verträgt sich diese Entwicklung mit einem nachhaltigen, unternehmerischen Handeln? Und: Wie können Kundinnen und Kunden erkennen, wie nachhaltig die Produkte oder Dienstleistungen eines Unternehmens sind? Über diese und weitere Fragen haben wir mit Dr. Rüdeger Köhler, Country Head Radiology Germany bei der Bayer Vital GmbH, gesprochen.

Herr Dr. Köhler, wie definieren Sie in Ihrem Unternehmen Nachhaltigkeit? Was sind aus Ihrer Sicht wichtige Arbeits- und Anwendungsbereiche?

Dr. Rüdeger Köhler: Die Bayer Pharma Geschäftseinheit Radiologie als Teil des Gesamtkonzerns ist den Zielen der Bayer $A G$ verpflichtet. Beiträge zu einer nachhaltigen Entwicklung zu leisten, ist ein zentrales
Element der Strategie und der Werte von Bayer. Im Sinne unserer Vision „Health for All, Hunger for None“ setzen wir uns für inklusives Wachstum und den verantwortungsbewussten Einsatz von Ressourcen ein. Dabei gehen für uns Nachhaltigkeit und wirtschaftlicher Erfolg Hand in Hand. Das heißt, unser Ziel ist es, mit nachhaltigen Lösungen wirtschaftlich erfolgreich zu sein. Dies bedeutet für uns auch, dass Nachhaltigkeit nicht „neben“ dem Geschäft betrieben wird, sondern unser ganzes Geschäft und all unsere Prozesse auf Nachhaltigkeit ausgerichtet werden. Indem wir an nachhaltigen Innovationen für die Landwirtschaft arbeiten, die dazu beitragen, dass ausreichend Nahrung für die wachsende Weltbevölkerung hergestellt werden kann - und das, ohne unsere Erde und ihre ökologischen Systeme zu überfordern. Und indem wir an innovativen Produkten von der Diagnose bis zur Behandlung arbeiten, um Krankheiten künftig besser erkennen, behandeln, vorbeugen oder sogar heilen zu können. Wie wichtig beides ist, hat uns das vergangene Jahr, das ganz im Zeichen der Pandemie stand, eindringlich vor Augen geführt.

Welchen Stellenwert hat Nachhaltigkeit in Ihrem Unternehmen und welche Ziele haben Sie für sich im Bereich Nachhaltigkeit definiert?

Als führendes Unternehmen in den Bereichen Gesundheit und Ernährung werden wir bei Bayer mit unseren Innovationen, Produkten und Dienstleistungen die Nachhaltigkeitsziele der Vereinten Nationen unterstützen und einige der grundlegenden Herausforderungen unserer Zeit adressieren. So haben wir etwa ein umfangreiches, konzernweites Dekarbonisierungsprogramm auf den Weg gebracht und wollen so dazu beitragen, die Erderwärmung auf $1,5^{\circ} \mathrm{C}$ zu begrenzen. Um unsere Emissionen bis Ende 2029 um mehr als 42 Prozent zu verringern, werden wir die Energieeffizienz 
an unseren Standorten steigern und beziehen unseren Strom dann zu 100 Prozent aus erneuerbaren Energien. Bis 2030 wollen wir ein klimaneutrales Unternehmen werden, indem wir alle noch verbleibenden Emissionen durch den Erwerb von Zertifikaten aus geprüften Klimaschutzprojekten kompensieren, die extern anerkannte Qualitätsstandards erfüllen.

Wie können Kundinnen und Kunden erkennen, wie nachhaltig die Produkte Ihres Unternehmens sind, zum Beispiel hinsichtlich Produktion, Nutzung und Entsorgung?

Das Wachstum und das zunehmende Altern der Weltbevölkerung sowie die steigende Belastung der natürlichen Ökosysteme stellen die Menschheit vor große Herausforderungen. Wir wollen maßgeblich dazu beitragen, Lösungen zu finden. Beispielsweise ist die Geschäftseinheit Radiologie mit ihrem Produktportfolio aus Kontrastmitteln, Kontrastmittelinjektoren und digitalen Lösungen an vorderster Front an der Seite der Anwenderinnen und Anwender, der Patientinnen und Patienten sowie der Gesundheitsversorgerinnen und -versorger in dem gemeinsamen Bemühen um noch nachhaltigere Angebote. Wir arbeiten aktiv daran, den Nutzerinnen und Nutzern von Kontrastmitteln Optionen zu einem nachhaltigeren Umgang mit Kontrastmittelrückständen im laufenden Betrieb anzubieten. Im Rahmen des re:contrast-Programms nehmen wir beispielsweise Produktreste von iodhaltigem Kontrastmittel von unseren Kundinnen und Kunden zurück und führen diese einem industriellen lod-Recyclingprozess zu. Durch diese Rückführung von lod in die Wertschöpfungsketten wird der Nutzungszyklus dieses aufwändig gewonnen Elements verlängert und Umwelteinträge minimiert.

Zum Portfolio Ihres Unternehmens gehören unter anderem auch Kontrastmittel. An welchen Lösungen arbeiten Sie, um zum Beispiel zu verhindern, dass Schwermetalle wie Gadolinium ins Trinkwasser gelangen?

Kontrastmittel, die bei der kontrastverstärkten medizinischen Bildgebung angewendet werden, unterstützen Ärztinnen und Ärzte bei der Diagnostik und Überwa- chung von Erkrankungen. Wir haben proaktiv gründliche Untersuchungen zum Umweltverhalten und zu Auswirkungen unserer iodbasierten Röntgenkontrastmittel und gadoliniumbasierten MRT-Kontrastmittel durchgeführt, um das mögliche Umweltverhalten der Rückstände beurteilen zu können. Diese Kontrastmittel wurden mit dem Ziel entwickelt, im menschlichen Körper keine pharmakologische Wirkung zu entfalten, sondern lediglich während der Körperpassage die radiologische Bildgebung zu verbessern oder sogar erst zu ermöglichen. Dafür ist die Stabilität des Kontrastmittels in vivo eine ganz zentrale Eigenschaft. Aufgrund dieser hohen Stabilität und der Ungiftigkeit von Kontrastmitteln ist es nicht verwunderlich, dass umfangreiche toxikologische und ökotoxikologische Untersuchungen keine Hinweise auf eine Gefährdung von Menschen oder Umwelt, zum Beispiel durch Vorhandensein als Spurenstoff in Oberflächengewässern ergeben haben. Der Verbleib von Arzneimitteln in der Umwelt wird zudem fortlaufend durch Behörden überprüft.

Zusätzlich zu beachten ist, dass aufgrund der immer höher entwickelten technischen Möglichkeiten, die uns heute zur Verfügung stehen, mittlerweile auch Kleinstmengen an Röntgenkontrastmittel in der Umwelt messbar sind und berichtet werden. Im Rahmen seiner Verantwortung als Hersteller beteiligt sich Bayer aktiv am sozialen und politischen Dialog mit verschiedenen Interessengruppen zu den potenziellen Umweltauswirkungen von Kontrastmittelrückständen. Hierzu zählen der Stakeholder-Dialog „Runder Tisch Röntgenkontrastmittel“, der Bestandteil der "Spurenstoffstrategie des Bundes" ist und vom - damaligen - Bundesministerium für Umwelt, Naturschutz und nukleare Sicherheit (BMU) initiiert wurde. Diesem gehören Branchenvertreterinnen und -vertreter (Originalherstellerinnen und -hersteller sowie Generika-Vertreiberinnen und -vertreiber von Kontrastmitteln), Verbraucherinnen und Verbraucher, Gesundheitsdienstleisterinnen und -dienstleister, Vertreterinnen und Vertreter der Abwasser- und Abfallbranche sowie Vertreterinnen und Vertreter von Nichtregierungsorganisationen (Umweltverbände und Zivilgesellschaft) an.

Ein wichtiges Ergebnis des Runden Tisches Röntgenkontrastmittel war die Durchfüh- rung und inhaltliche Begleitung einer Konzeptionsstudie, die die Einführung von Urinsammelsystemen für die Reduktion des Eintrags von Röntgenkontrastmitteln ins Abwasser über die Patientinnen und Patienten umfänglich bewertet und Lösungswege aufgezeigt hat. Der Bericht der Studie liegt dem Runden Tisch und der Öffentlichkeit vor - nächste Schritte sind im StakeholderDialog-Prozess in Arbeit.

In der Medizin und insbesondere in der Radiologie sind die Innovationszyklen sehr kurz. Wie lässt sich der „Innovationszwang“ mit dem Ziel der Nachhaltigkeit vereinbaren?

Da wir unser Geschäft konsequent auf Nachhaltigkeit ausrichten, prüfen wir auch bei Innovationen, wie sie zu unseren Nachhaltigkeitszielen beitragen. Wir arbeiten an Lösungen für möglichst effiziente Arbeitsabläufe und Kontrastmittelgaben. Mit immer innovativeren Produkten und Technologien stellt die medizinische Bildgebung einen wichtigen Treiber für die Umgestaltung der Gesundheitsversorgung, die Ausweitung der personalisierten Medizin und die Verbesserung der Behandlungsergebnisse dar. Innovationen spielen daher eine wichtige Rolle, um die Effizienz im Gesundheitswesen zu steigern. Darüber hinaus leisten Innovationen in der Bildgebung einen wichtigen Beitrag für die Nachhaltigkeit des Gesundheitssystems allgemein: Die medizinische Bildgebung ist sowohl für die Bestätigung und Beurteilung vieler Erkrankungen als auch für die Überwachung des Behandlungserfolgs von entscheidender Bedeutung. Zudem sind unsere Produkte so gestaltet, dass sie eine lange Lebensdauer aufweisen und über die Zeit weiter aufgerüstet werden können.

Was können wir für nachhaltige Innovationen von Ihrem Unternehmen in den nächsten Jahren erwarten?

Wir haben viele Schritte unternommen, um bei den aktuellen technologischen Entwicklungen in den Life Sciences ganz vorne dabei zu sein und mit unseren Innovationen zu einem nachhaltigen Gesundheitssystem beizutragen. Atemberaubende Fortschritte in der Zellbiologie und bei der Genom-Editierung und immer spezifischere Applikationstechnologien revolutionieren die Life 
Sciences und schaffen - teilweise in Verbindung mit Informationstechnologie und künstlicher Intelligenz (KI) - ungeahnte neue Möglichkeiten für Gesundheit und Ernährung. Diese Biorevolution wollen wir entscheidend mitgestalten. Für den Bereich Radiologie werden beispielsweise digitale Technologien eine wichtige Rolle spielen: Künstliche Intelligenz kann nachhaltig dazu beitragen, Ressourcen gezielter einzusetzen, Diagnosen früher zu stellen und die Anzahl von Fehldiagnosen zu verringern. Dadurch können viele Patientinnen und Patienten früher mit einer passsenden Behandlung beginnen.

Wir arbeiten zum Beispiel an einem digitalen kuratierten Marktplatz für medizinische Bildgebung: eine Art App Store für Radiolo- ginnen und Radiologen und ihre Teams, der Zugang zu hochwertigen digitalen Anwendungen für die klinische Bildgebung und Arbeitsabläufe einschließlich KI-fähiger Lösungen bietet. Angesichts des immer größer werdenden Marktes an bildgebenden Anwendungen und mangelnder finanzieller und zeitlicher Ressourcen und IT-Expertise können Radiologinnen und Radiologen üblicherweise nur eine kleine Anzahl neuer Software-Anwendungen nutzen. Der kuratierte Marktplatz wird es den Radiologinnen und Radiologen und ihren Teams ermöglichen, eine eigene Auswahl an bildgebenden, geprüften Anwendungen zusammenzustellen und schneller und besser in ihre Abläufe zu integrieren. Beispielsweise wird ihnen eine radiologische KI-Lösung zur Verfügung stehen, die bei der Diagnos- tik und Priorisierung von Patientinnen und Patienten mit Schlaganfall in der Notfallsituation hilft, um die Zeit bis zur Behandlung zu verkürzen.

Eine andere Lösung unterstützt die behandelnde Ärztin und den behandelnden Arzt bei der Früherkennung von kleinen Rundherden in der Lunge, die auf eine schwere Erkrankung, wie beispielsweise Lungenkrebs, hinweisen können. Mit all diesen Maßnahmen im Sinne von „Science for a better life“ tragen wir dazu bei, die großen Herausforderungen unserer Zeit anzugehen und die gesellschaftliche Wirkung unseres Unternehmens und unseres Geschäfts zu stärken. 